\title{
Associação entre maus-tratos familiares e excesso de peso e de gordura em escolares do município do Rio de Janeiro/RJ, Brasil
}

\author{
Association between domestic maltreatment and excess weight \\ and fat among students of the city/state of Rio de Janeiro, Brazil
}

Ana Maria Vieira Lourenço da Silva ${ }^{1}$

Maria Helena Hasselmann ${ }^{2}$

${ }^{1}$ Policlínica Hélio Pellegrino, Secretaria Municipal de Saúde do Rio de Janeiro. R. Matoso 125, Praça da Bandeira. 20270132 Rio de Janeiro RJ Brasil.amvls@hotmail.com ${ }^{2}$ Depto. Nutrição Social, Instituto de Nutrição, Universidade do Estado do Rio de Janeiro. Rio de Janeiro RJ Brasil.

\begin{abstract}
This study sought to investigate the relationship between domestic maltreatment and excess weight, body fat and abdominal fat among adolescents from the public schools in the city of Rio de Janeiro - Brazil. It involved a cross-sectional study using data from the Risk Factor Surveillance System and Protection of Health of Adolescents, held in 2007 by the municipality of Rio de Janeiro. The sample consisted of 1628 students aged 13 to 19 years, of both sexes, in the 9 th year of school. Excess weight, body fat and abdominal fat (outcome variables) and domestic maltreatment (physical and verbal aggression) were evaluated. The association between domestic maltreatment and outcome variables was conducted via logistic regression models. Domestic maltreatment showed an inverse relationship to excess weight and fat for girls, but only physical aggression was significantly associated with excess weight $(O R=$ $0.499, C I=0.212$ to 0.951). As for the boys, the results pointed to excess weight and fat but without statistical significance. Health policies must consider the differences between the genders in coping with experiences of violence that may influence the health and well-being of adolescents.

Key words Domestic violence, Adolescent, Obesity, Abdominal fat, Adiposity
\end{abstract}

Resumo O objetivo deste estudo foi investigar a relação entre maus-tratos na família e excesso de peso, de gordura corporal e de gordura abdominal dos adolescentes da rede pública de ensino de município do Rio de Janeiro - Brasil. Trata-se de um estudo transversal que utiliza dados do Sistema de Vigilância de Fatores de Risco e Proteção à Saúde de Adolescentes, realizado em 2007, pelo município do Rio de Janeiro. A amostra foi composta por 1.628 escolares de 13 a 19 anos, de ambos os sexos, cursando o $9^{\circ}$ ano do ensino fundamental. $O$ excesso de peso, de gordura corporal e de gordura abdominal (desfechos) e os maus-tratos familiares (a agressão física e verbal) foram avaliados. A associação entre os maus-tratos e os desfechos foi realizada via modelos de regressão logística. Os maus-tratos apresentaram uma relação inversa com o excesso de peso e o de gordura para as meninas, mas somente a agressão fisica se associou significativamente com o excesso de peso $(R C=$ 0,499; IC =0,212-0,951). Já para os meninos, os resultados apontaram para o excesso de peso e de gordura mas sem significância estatística. Políticas de saúde devem considerar as diferenças existentes entre os gêneros no enfrentamento às experiências de violência que possam influenciar a saúde e o bem-estar do adolescente.

Palavras-chave Violência doméstica, Adolescente, Obesidade, Gordura abdominal, Adiposidade 


\section{Introdução}

O excesso de peso na infância e na adolescência é um problema de saúde pública que vem crescendo na maioria dos países do mundo ${ }^{1}$. No Brasil, inquéritos populacionais também têm detectado esse aumento em adolescentes ${ }^{2,3}$, o que causa grande preocupação uma vez que o excesso de peso predispõe às doenças cardiovasculares como hipertensão $0^{4}$, dislipidemia ${ }^{5}$, alterações no metabolismo de glicose $e^{6}$, bem como a ocorrência de síndrome metabólica ${ }^{7}$ que muitas vezes se inicia na infância e na adolescência ${ }^{8}$.

Em paralelo a isso, a violência familiar vem se tornando uma questão social relevante ${ }^{9}$ e as crianças e os adolescentes são apontados como vítimas que apresentam grande dificuldade de revelar os abusos sofridos ${ }^{10}$. São inúmeras as consequências da violência familiar na saúde dos adolescentes, entre elas, podem-se citar as psicológicas (depressão e ansiedade), as comportamentais, as relativas ao processo de aprendizagem, aquelas relacionadas aos resultados negativos de saúde física ${ }^{11}$, assim como as ligadas ao abuso de substâncias ilícitas e ao risco de doenças sexualmente transmissíveis ${ }^{12}$.

Investigar a violência familiar como um possível fator associado ao excesso de peso (sobrepeso e obesidade) em adolescentes é algo recente e pouco explorado ${ }^{13}$. A maioria da literatura sobre o tema se refere a estudos realizados em outros países, no Brasil somente duas investigações foram identificadas ${ }^{14,15}$. Ressalta-se que grande parte das pesquisas utiliza o índice de massa corporal (IMC) como o método de classificação do sobrepeso e da obesidade ${ }^{16}$. Apesar do IMC ser considerado como uma medida importante para se detectar a ocorrência de sobrepeso e obesidade, não distingue os diferentes tipos de tecidos (gordura, osso ou músculo) em relação aos seus excessos ou à sua distribuição corpórea ${ }^{4}$. Nesse sentido, pesquisas que utilizam outras técnicas para identificação do excesso de peso e de gordura como a estimativa de gordura corporal por meio das dobras cutâneas para classificar o excesso de gordura corporal ${ }^{17} \mathrm{e}$ a razão cintura/altura (RCA) para a gordura abdominal ${ }^{18}$ são bem-vindas. Os estudos existentes sobre o assunto apontam para uma associação entre os maus-tratos na infância e a ocorrência de excesso de peso em adultos, mas quando se avalia adolescentes esta relação parece não acontecer. Ressalta-se ainda que a literatura mostra resultados contraditórios - associação ${ }^{10}$ e não associação ${ }^{16}$ - entre os maustratos e o excesso de peso.
Portanto, explorar a associação entre violência na família e a ocorrência do excesso de peso e de gordura é de suma importância para a construção de um novo olhar dos profissionais de saúde, de educação e do público em geral para o problema $^{4}$. O objetivo deste estudo foi investigar a associação entre os maus-tratos familiares e o excesso de peso, de gordura corporal e de gordura abdominal em adolescentes.

\section{Métodos}

\section{Fonte de dados}

Trata-se de um estudo transversal com dados secundários extraídos do inquérito - Sistema de Vigilância de Fatores de Risco e Proteção à Saúde de Adolescentes - realizado em 2007 com escolares que cursavam o $9^{\circ}$ ano do ensino fundamental de escolas públicas do município do Rio de Janeiro. Este sistema de vigilância foi inspirado no sistema de vigilância europeu, Health Behaviour in School-aged Children (HBSC), e no sistema americano, Youth Risk Behavior Surveillance System (YRBSS) do Center for Disease Control and Prevention (CDC). Mais informações sobre conceito e implementação do sistema de vigilância encontram-se disponíveis em outra publicação ${ }^{19}$.

No ano de 2007, a rede pública possuía 1082 escolas e 743.416 alunos distribuídos por 10 Coordenadorias Regionais de Educação (CRE). Deste total, 365 escolas possuíam 43.099 alunos matriculados no último ano do ensino fundamental, representando cerca de $6 \%$ do total de matrículas. Para a realização deste inquérito foi adotado um desenho amostral complexo, que teve por objetivo selecionar dados representativos correspondentes aos alunos matriculados no nono ano do ensino fundamental das escolas públicas municipais do Rio de Janeiro. Como no ano de 2007, os inquéritos municipais investigavam cerca de 1000 a 1800 estudantes, e considerando os recursos disponíveis, optou-se por selecionar 1700 estudantes, de 170 turmas e de 136 escolas. As escolas foram divididas em 10 estratos de acordo com as regiões (CRE) e, como havia duas unidades de amostragem (turmas e alunos), foram realizados sorteios em dois estágios: 1) dentro de cada estrato natural (CRE), foi feito o sorteio das turmas com probabilidade proporcional ao tamanho delas, conforme o número de alunos contidos em cada turma, e 2) os alunos elegíveis de cada turma selecionada foram sorteados por meio de amostra aleatória simples, com 
tamanho pré-definido de 10 alunos por turma. Os alunos elegíveis que concordaram em participar do inquérito, tinham que apresentar o Termo de Consentimento Livre e Esclarecido (TCLE) assinado pelos responsáveis, que estar presentes no dia da coleta de dados na escola e responder ao questionário e participar da avaliação antropométrica. Destes alunos, 52 não foram avaliados por não estarem presentes no dia agendado para coleta de dados ou por não apresentarem o TCLE assinado pelos responsáveis, totalizando 1648 escolares $^{20}$. Foram excluídos 16 adolescentes por não informarem o sexo e a idade ${ }^{20}$ e 4 alunos por apresentarem idade superior a 19 anos, uma vez que este estudo aborda escolares na fase da adolescência ${ }^{21}$. Assim, a amostra final compreendeu 1628 adolescentes.

Empregou-se um questionário autoaplicável e anônimo com os alunos do inquérito. Para a realização deste estudo foram utilizadas do questionário as questões/informações relativas às características sociodemográficas; à atividade física; aos hábitos sedentários; ao consumo de cigarros, álcool e outras drogas; à segurança; à imagem corporal; e às relações com a família, amigos e escola.

O estudo foi aprovado e autorizado pelo Comitê de ética da Secretaria Municipal de Saúde e Defesa Civil do Rio de Janeiro.

\section{Variáveis do estudo}

\section{Desfechos - excesso de peso, excesso de gordura corporal e excesso de gordura abdominal}

As medidas antropométricas foram utilizadas na aferição do excesso de peso, do excesso de gordura corporal e do excesso de gordura abdominal. Os pesquisadores (antropometristas) foram treinados e suas medidas foram padronizadas segundo as técnicas de Lohman ${ }^{22}$ e Habit$\mathrm{ch}^{23}$. Foram utilizados os seguintes equipamentos para a mensuração das medidas: 1) balança solar portátil (TANITA ${ }^{\circledR}$ ) com capacidade de $150 \mathrm{~kg}$ e precisão de $0,2 \mathrm{~kg}$ para o peso corporal; 2) estadiômetro portátil (AlturExata ${ }^{\circledR}$ ) com escala principal em centímetros e precisão de $0,1 \mathrm{~cm}$ para a altura; 3 ) adipômetro Cescorf com precisão de $0,1 \mathrm{~cm}$ para as dobras cutâneas tricipital e subescapular; e 4) fita métrica elástica com precisão de $0,1 \mathrm{~mm}$ para o perímetro abdominal (circunferência de cintura).

A classificação do excesso de peso foi realizada por meio do IMC - peso/altura ${ }^{2}\left(\mathrm{~kg} / \mathrm{m}^{2}\right)$ - a partir de critérios de percentis segundo sexo e faixa etária estabelecidos pela Organização Mundial de Saúde (OMS) ${ }^{24}$ para sobrepeso e obesidade. Foram considerados com excesso de peso os adolescentes com IMC > percentil 85 .

Para se obter o excesso de gordura corporal, primeiramente foi calculado o percentual de gordura corporal por meio da fórmula proposta por Slaughter et al. ${ }^{25}$ para dobras cutâneas tricipital e subescapular segundo o sexo, a maturação sexual e raça. Posteriormente, a estimativa de gordura corporal foi classificada pelos critérios de Lohman $^{26}$ em para excesso de gordura corporal nas meninas que apresentassem \% de gordura corporal $>25$ e nos meninos $>20$.

A gordura abdominal foi avaliada pela razão cintura/altura (RCA). Este índice foi calculado como a proporção entre o perímetro abdominal $(\mathrm{cm})$ e a altura $(\mathrm{cm})$. Os adolescentes que apresentavam RCA $>0,5$ foram classificados como tendo excesso de gordura abdominal ${ }^{4}$.

\section{Exposições - agressão física e verbal}

No presente estudo considerou-se como maus-tratos familiares a agressão física e verbal. A agressão verbal foi avaliada por meio da questão: "Nos últimos 30 dias, com que frequência sua família te esculachou (tratou muito mal)?” e apresentava cinco opções de respostas, tendo como referência os últimos 30 dias a saber: (a) nenhuma vez; (b) raramente; (c) às vezes; (d) na maior parte das vezes; e (e) sempre. A agressão física foi avaliada pela questão "Nos últimos 30 dias, quantas vezes você foi agredido fisicamente por um adulto da família?" e as respostas variavam conforme o número de vezes com referência aos últimos 30 dias: (a) Nenhuma vez, (b) 1 (uma) vez, (c) 2 ou 3 vezes, (d) 4 ou 5 vezes, (e) 6 ou 7 vezes, (f) 8 ou 9 vezes, (g) 10 ou 11 vezes, e (h) 12 vezes ou mais. Para análise estatística, a agressão verbal foi categorizada como: presente (raramente a sempre) e ausente (nenhuma vez) e a agressão física também foi classificada como: presente (uma vez ou mais) e ausente (nenhuma vez).

Foi realizado estudo de confiabilidade com 208 estudantes (12,4\% do total estudado), pertencentes a 25 turmas de todas CREs. O questionário foi reaplicado 7 a 15 dias após a primeira visita à escola, e a confiabilidade avaliada por meio de estimativa de kappa (k), k ajustada para a prevalência (quando a prevalência foi superior a $80 \%$ e inferior a 20\%) e k com ponderação quadrática (para variáveis ordinais). As perguntas relativas à agressão física e à verbal estão compreendidas nos módulos pertinentes à segurança e à relação com a família, amigos e escola e apresen- 
taram nível de confiabilidade, respectivamente, de 0,72 (bom) e de 0,54 (razoável) ${ }^{20}$.

\section{Covariáveis}

Foram usadas informações socioeconômicas e características dos adolescentes como idade em anos, sexo, raça/cor - negra e não negra -, escolaridade do chefe de família - classificada em ensino fundamental incompleto, fundamental completo e médio completo - e escores de bens - construído pelo somatório simples de itens presentes em domicílio (telefone fixo, aparelho DVD, forno de micro-ondas, computador, carro, moto, empregada doméstica em pelo menos cinco dias da semana), classificados por pesos que variaram de 0 (nenhum bem ou serviço) a 7 (todos os bens e serviço previsto) e posteriormente agrupados por terços da distribuição observada na amostra em alto (possui 5 a 7 bens), médio (possui 3 a 4 bens) e baixo (possui nenhum até 2 bens).

As atividades sedentárias foram avaliadas em função do tempo gasto assistindo TV ou jogando videogame ou usando o computador em um dia de semana comum. Elas foram categorizadas de acordo com a recomendação da American Academy of Pediatrics ${ }^{27} \mathrm{em}: \leq 2$ horas/dia (não sedentários) e $>2$ horas/dia (sedentários);

A Atividade física foi analisada com base nas informações referentes à prática de atividades de lazer (orientada ou livre), aos deslocamentos ativos para a escola (caminhada e uso da bicicleta) e as atividades realizadas na escola (com e sem o professor) nos sete dias que antecederam o estudo, somados o tempo em minutos. Os escolares foram classificados em ativos (> 300 minutos/ semana) e insuficientemente ativos $(\leq 300 \mathrm{minu}-$ tos/semana) $)^{28}$;

As covariáveis construídas com base nas perguntas relativas à percepção do escolar sobre a relação com a família e com a escola; sobre o consumo de cigarros, álcool e outras drogas e sobre a imagem corporal foram: 1) Família ciente do tempo livre - "Nos últimos 30 dias, com que frequência seus pais ou responsáveis sabiam realmente o que você estava fazendo em seu tempo livre?" - não (nenhuma) e sim (raramente a sempre); 2) Pais cientes de faltar aula - "Nos últimos 30 dias, em quantos dias você faltou às aulas ou à escola sem permissão dos seus pais ou responsáveis?" - não (nenhuma) e sim (uma ou mais vezes); 3 ) Elogiado pela família - "Nos últimos 30 dias, com que frequência sua família te elogiou?" - não (nenhuma vez) e sim (raramente a sempre); 4) Pais se importam com o uso de fumo/álcool/droga - bebida alcóolica ("Se você chegasse em casa bêbedo, qual seria a reação da família se ela ficasse sabendo?"), fumo ("Se você fumasse cigarros, qual seria a reação da família se ela ficasse sabendo?") e maconha ("Se você fumasse maconha, qual seria a reação da família se ela ficasse sabendo?") - não se importam e se importam; 5) Diálogo com o professor - "Nos últimos 30 dias, com que frequência você conversou com seus professores sobre assuntos que não tinham relação com a aula? - não (nenhuma vez) e sim (raramente a sempre); 6) Boa relação com colega - Nos últimos 30 dias, com que frequência os colegas de escola trataram você bem e/ou foram legais com você?" - não (Nenhuma vez) e sim (raramente a sempre); 7) Descrição do peso - "Como você descreve seu corpo?" - abaixo, peso certo ou acima; 8) Realização de algum procedimento em relação ao peso - "O que você está fazendo em relação ao seu peso?” - não (não estou fazendo nada) e sim (estou tentando perder, ganhar ou manter o mesmo peso); 9) Satisfação com o peso - "Nos últimos 30 dias, com que frequência você se sentiu feliz com seu corpo" - satisfeito (às vezes até sempre) e insatisfeito (nenhuma vez ou raramente); 10) Realização de algum procedimento em relação ao peso - "Nos últimos 30 dias, você vomitou ou tomou laxantes para perder peso ou evitar ganhar peso?" - não e sim; e 11) Realização de práticas extremas para controle do peso - "Nos últimos 30 dias, você tomou algum comprimido, líquido ou pó para perder ou manter peso sem acompanhamento médico?" - não e sim.

\section{Análises estatísticas}

Em primeiro lugar, foram realizadas análises univariadas, determinando frequências (prevalências) para variáveis categóricas, e medida-sumário (média e erro padrão) para variáveis numéricas. Desde que os achados anteriores sugeriram possíveis diferenças de sexo na associação entre maus-tratos e o excesso de peso ${ }^{29}$, todas as análises foram elaboradas para sexo masculino e sexo feminino separadamente. Para as variáveis categóricas utilizou-se o teste Chi-quadrado e para as variáveis numéricas, o teste t de Student não pareado.

Foram realizadas análises de regressão logística univariada (bruta) entre os desfechos, as variáveis de exposição e as covariáveis de interesse. Para se verificar a associação entre os maus-tratos e os desfechos, foram criados modelos logísticos multivariados (ajustados) entre cada variável de 
exposição (maus-tratos familiares) e os desfechos (excesso de peso, excesso de gordura corporal e excesso de gordura abdominal) ajustados pelas covariáveis que apresentassem $\mathrm{p}$-valor $<0,20$ nas análises brutas. Considerou-se o p-valor $<0,05$ como critério de significância estatística para permanência de variáveis nos modelos logísticos multivariados. Estes modelos foram diagnosticados pelo teste de Hosmer-Lemeshow que concebeu como modelo bem ajustado aquele que apresentasse $\mathrm{p}$-valor $>0,05$. Os odds ratios (OR) de cada variável de exposição nos modelos logísticos multivariados foram apresentados, além dos respectivos intervalos de 95\% de confiança e p-valores.

Como o desenho da amostra era do tipo complexo, todos os dados de prevalência, $\mathrm{OR}$, e intervalos de confiança de 95\% foram ponderados. Os tamanhos das amostras foram relatados em sua forma não ponderada.

As análises estatísticas foram realizadas com o auxílio dos Programas SPSS, versão 21.0 e Stata 13.1 para Mac.

\section{Resultados}

A amostra do estudo compreendeu 1628 adolescentes, sendo $730(44,2 \%)$ meninos e 898 $(55,8 \%)$ meninas, com idade média de 14,93 anos $(\mathrm{EP}=0,05)$. A Tabela 1 descreve as características da população estudada. Entre elas, destaca-se que aproximadamente $27 \%$ deles eram in- satisfeitos com o seu peso, sendo esta insatisfação quase duas vezes maior para as meninas ( $\mathrm{p}$-valor $<0,001$ ); que entre os adolescentes estudados mais de 50\% deles estavam realizando algum procedimento para modificar o seu peso, sendo mais presente para o sexo feminino ( $\mathrm{p}$-valor =0,030); e que as meninas também eram duas vezes mais propensas a realizar práticas extremas para controle de peso $(\mathrm{p}$-valor $=0,003)$. Foi notado que cerca de $18 \%$ deles apresentavam excesso de peso, $8 \%$ gordura abdominal aumentada e $36,5 \%$ excesso de gordura corporal. A gordura corporal foi a única medida a apresentar distinção entre os sexos - maior percentual de gordura para as meninas ( $\mathrm{p}$-valor $=0,002)$. Com relação aos maus-tratos familiares, $36 \%$ dos estudantes apresentaram agressão verbal e 8,5\% com a física. Ao se fazer a distinção por sexo no que tange aos maus-tratos, observou-se uma maior prevalência de agressão física (10,2\% versus 6,4\%, p-valor < $0,001)$ e verbal $(40,6 \%$ versus $29,7 \%$, p-valor $=$ $0,005)$ para as meninas.

A Tabela 2 apresenta as análises entre as variáveis e os diferentes desfechos para o sexo feminino. Houve diferença estatisticamente significativa para escolaridade do chefe de família ( $p$-valor $=0,034)$ e escores de bens $(p$-valor $=0,035)$ com relação ao excesso de peso. As meninas apresentavam cerca de 0,6 menos chances de ter excesso de peso quando tinham baixo poder aquisitivo e chefe de família com baixa escolaridade. Já o excesso de gordura corporal e o excesso de gordura abdominal não apresentaram valores estatistica-

Tabela 1. Características, informações ambientais, demográficas e sociais, segundo população total e sexo. Rede Pública Municipal do Rio de Janeiro, 2007.

\begin{tabular}{|c|c|c|c|c|c|c|}
\hline \multirow{2}{*}{ Variáveis categóricas } & \multicolumn{2}{|c|}{ População total } & \multicolumn{2}{|c|}{ Sexo masculino } & \multicolumn{2}{|c|}{ Sexo feminino } \\
\hline & $\mathbf{N}$ & $\%$ & $\mathbf{N}$ & $\%$ & $\mathbf{N}$ & $\%$ \\
\hline Cor/raça & 1612 & 100 & 721 & 44,1 & 891 & 55,9 \\
\hline Negra & 240 & 14,7 & 121 & 16,3 & 119 & 13,4 \\
\hline Não negra & 1372 & 85,3 & 600 & 83,7 & 772 & 86,6 \\
\hline Escores de bens ${ }^{\star}$ & 1601 & 100 & 721 & 44,4 & 880 & 55,6 \\
\hline Baixo poder aquisitivo & 545 & 33,8 & 224 & 30,3 & 321 & 36,6 \\
\hline Médio poder aquisitivo & 747 & 46,7 & 339 & 47,1 & 408 & 46,4 \\
\hline Alto poder aquisitivo & 309 & 19,5 & 158 & 22,6 & 151 & 16,9 \\
\hline Escolaridade do chefe da família & 1490 & 100 & 672 & 44,4 & 818 & 55,6 \\
\hline Fundamental incompleto & 690 & 46 & 292 & 42,9 & 398 & 48,5 \\
\hline Fundamental completo & 490 & 33,1 & 226 & 34 & 264 & 36,3 \\
\hline Médio completo & 310 & 20,9 & 154 & 23,1 & 156 & 19,2 \\
\hline Elogiado pela família & 1598 & 100 & 713 & 44 & 885 & 56 \\
\hline Não & 246 & 15,6 & 115 & 16,3 & 131 & 15 \\
\hline Sim & 1352 & 84,4 & 593 & 83,7 & 754 & 85 \\
\hline
\end{tabular}


Tabela 1. Características, informações ambientais, demográficas e sociais, segundo população total e sexo. Rede Pública Municipal do Rio de Janeiro, 2007.

\begin{tabular}{|c|c|c|c|c|c|c|}
\hline \multirow{2}{*}{ Variáveis categóricas } & \multicolumn{2}{|c|}{ População total } & \multicolumn{2}{|c|}{ Sexo masculino } & \multicolumn{2}{|c|}{ Sexo feminino } \\
\hline & $\mathbf{N}$ & $\%$ & $\mathbf{N}$ & $\%$ & $\mathbf{N}$ & $\%$ \\
\hline Família ciente do tempo livre & 1596 & 100 & 708 & 43,7 & 888 & 56,3 \\
\hline Não & 389 & 24,6 & 195 & 27,9 & 194 & 22 \\
\hline Sim & 1207 & 75,4 & 513 & 72,1 & 694 & 78 \\
\hline Pais ciente de faltar aula & 1599 & 100 & 711 & 43,8 & 888 & 56,2 \\
\hline Não & 1157 & 72,6 & 519 & 73,3 & 638 & 72 \\
\hline Sim & 442 & 27,4 & 192 & 26,7 & 250 & 28 \\
\hline Pais se importam com uso de fumo/álcool/droga & 1624 & 100 & 728 & 44,2 & 896 & 55,8 \\
\hline Não se importam & 117 & 7,4 & 56 & 7,8 & 61 & 7,2 \\
\hline Se importam & 1507 & 92,6 & 672 & 92,2 & 835 & 92,8 \\
\hline Boa relação com colega ${ }^{\star}$ & 1596 & 100 & 711 & 43,9 & 885 & 56,1 \\
\hline Não & 91 & 5,7 & 58 & 8,3 & 33 & 3,7 \\
\hline Sim & 1505 & 94,3 & 653 & 91,7 & 852 & 96,3 \\
\hline Diálogo com o professor & 1613 & 100 & 720 & 44 & 893 & 56 \\
\hline Não & 921 & 57,3 & 408 & 57 & 513 & 57,5 \\
\hline Sim & 692 & 42,7 & 312 & 43 & 380 & 42,4 \\
\hline Satisfação com o peso ${ }^{*}$ & 1596 & 100 & 711 & 43,9 & 885 & 56,1 \\
\hline Satisfeito & 1162 & 72,7 & 560 & 78,2 & 602 & 68,5 \\
\hline Insatisfeito & 434 & 27,3 & 151 & 21,8 & 283 & 31,5 \\
\hline Descrição do peso* & 1608 & 100 & 718 & 44 & 890 & 56 \\
\hline Peso certo & 704 & 43,8 & 353 & 49,3 & 351 & 39,5 \\
\hline Abaixo do peso & 375 & 23,2 & 223 & 30,9 & 152 & 17,2 \\
\hline Acima do peso & 529 & 33 & 142 & 19,8 & 387 & 43,3 \\
\hline Realização de algum procedimento em relação ao peso* & 1615 & 100 & 721 & 44 & 894 & 56 \\
\hline Não & 642 & 39,5 & 309 & 42,5 & 333 & 37,2 \\
\hline Sim & 973 & 60,5 & 412 & 57,5 & 561 & 62,8 \\
\hline Realização de práticas extremas para controle do peso* & 1606 & 100 & 716 & 43,9 & 890 & 56,1 \\
\hline Não & 1512 & 94 & 688 & 96,1 & 824 & 92,4 \\
\hline Sim & 94 & 6 & 28 & 3,94 & 66 & 7,6 \\
\hline Atividade física* & 1578 & 100 & 697 & 43,6 & 881 & 56,4 \\
\hline Ativo & 508 & 31,7 & 323 & 46,3 & 185 & 20,5 \\
\hline Insuficientemente ativo & 1070 & 68,3 & 374 & 56,7 & 696 & 79,5 \\
\hline Atividades Sedentárias & 1610 & 100 & 717 & 43,9 & 893 & 56,1 \\
\hline Não sedentário & 163 & 10,1 & 67 & 9,1 & 96 & 10,9 \\
\hline Sedentário & 1447 & 89,9 & 650 & 90,9 & 797 & 89,1 \\
\hline Excesso de gordura abdominal & 1628 & 100 & 730 & 44,2 & 898 & 55,8 \\
\hline Sem excesso & 1496 & 91,9 & 681 & 93,2 & 815 & 90,9 \\
\hline Com excesso & 132 & 8,1 & 49 & 6,8 & 83 & 9,1 \\
\hline Excesso de peso & 1594 & 100 & 709 & 43,8 & 885 & 56,2 \\
\hline Sem excesso & 1314 & 82,3 & 593 & 83,5 & 721 & 81,4 \\
\hline Com excesso & 280 & 17,7 & 116 & 16,5 & 164 & 18,6 \\
\hline Excesso de gordura corporal ${ }^{\star}$ & 1619 & 100 & 721 & 43,9 & 898 & 56,1 \\
\hline Sem excesso & 1025 & 63,5 & 491 & 68 & 534 & 60 \\
\hline Com excesso & 594 & 36,5 & 230 & 32 & 364 & 40 \\
\hline Agressão física* & 1621 & 100 & 776 & 44,2 & 895 & 55,8 \\
\hline Não & 1485 & 91,5 & 679 & 93,6 & 806 & 89,8 \\
\hline Sim & 136 & 8,5 & 47 & 6,4 & 89 & 10,2 \\
\hline Agressão verbal ${ }^{\star}$ & 1600 & 100 & 713 & 43,9 & 887 & 56,1 \\
\hline Não & 1029 & 64,2 & 500 & 70,3 & 529 & 59,4 \\
\hline Sim & 571 & 35,8 & 213 & 29,7 & 358 & 40,6 \\
\hline
\end{tabular}

Teste qui-quadrado de yale. ${ }^{*}$-valor $<0,05$ 
Tabela 2. Regressão logística univariada entre os desfechos e as variáveis. Adolescentes do sexo feminino. Rede Pública Municipal do Rio de Janeiro, 2007.

\begin{tabular}{|c|c|c|c|c|c|c|}
\hline \multirow{2}{*}{ Variáveis } & \multicolumn{2}{|c|}{ EP } & \multicolumn{2}{|r|}{ EG } & \multicolumn{2}{|c|}{ EGA } \\
\hline & OR & IC & OR & IC & OR & IC \\
\hline Idade & 0,930 & $0,793-1,090$ & 1,034 & $0,913-1,171$ & 0,942 & $0,760-1,166$ \\
\hline \multicolumn{7}{|l|}{ Cor/raça } \\
\hline Negra & 0,916 & $0,522-1,609$ & 0,790 & $0,519-1,204$ & 0,969 & $0,464-2,021$ \\
\hline Não negra & 1 & & 1 & & 1 & \\
\hline \multicolumn{7}{|l|}{ Escolaridade do chefe de família } \\
\hline Fundamental incompleto & $0,598^{\star * *}$ & $0,369-0,970$ & $0,777^{\star}$ & $0,531-1,137$ & $0,590^{* *}$ & $0,328-1,061$ \\
\hline $\begin{array}{l}\text { Fundamental completo e médio } \\
\text { incompleto }\end{array}$ & 0,918 & $0,576-1,465$ & 1,081 & $0,732-1,597$ & $0,624^{*}$ & $0,336-1.161$ \\
\hline Médio incompleto & 1 & & 1 & & 1 & \\
\hline \multicolumn{7}{|l|}{ Escores de bens } \\
\hline Baixo poder aquisitivo & $0,582^{\star * \star}$ & $0,346-0,980$ & 1,098 & $0,728-1,566$ & 1,053 & $0,488-2,272$ \\
\hline Médio poder aquisitivo & $0,632^{\star *}$ & $0,385-1,038$ & 1,108 & $0,771-1,592$ & 1,324 & $0,620-2,829$ \\
\hline Alto poder aquisitivo & 1 & & 1 & & 1 & \\
\hline \multicolumn{7}{|l|}{ Elogiado pela família } \\
\hline Não & 0,932 & $0,590-1,470$ & 0,856 & $0,587-1,257$ & 0,746 & $0,379-1,470$ \\
\hline Sim & 1 & & 1 & & 1 & \\
\hline \multicolumn{7}{|l|}{ Família ciente do tempo livre } \\
\hline Não & 1,159 & $0,807-1,665$ & $0,730^{* *}$ & $0,511-1,044$ & 0,968 & $0,588-1,594$ \\
\hline Sim & 1 & & 1 & & 1 & \\
\hline \multicolumn{7}{|l|}{ Pais cientes de faltar aula } \\
\hline Não & 1,174 & $0,827-1,668$ & $1,209^{*}$ & $0,908-1,608$ & 1,117 & $0,681-1,831$ \\
\hline Sim & 1 & & 1 & & 1 & \\
\hline \multicolumn{7}{|l|}{$\begin{array}{l}\text { Pais se importam com uso de álcool/ } \\
\text { fumo/drogas }\end{array}$} \\
\hline Não se importam & 0,909 & $0,461-1,791$ & 0,962 & $0,592-1,563$ & 0,910 & $0,349-2,369$ \\
\hline Se importam & 1 & & 1 & & 1 & \\
\hline \multicolumn{7}{|l|}{ Boa relação com colega } \\
\hline Não & 0,785 & $0,303-2,037$ & 0,721 & $0,352-1,478$ & $0,253^{*}$ & $0,033-1,917$ \\
\hline Sim & 1 & & 1 & & 1 & \\
\hline \multicolumn{7}{|l|}{ Diálogo com o professor } \\
\hline Não & 0,983 & $0,693-1,396$ & 1,082 & $0,818-1,432$ & 1,101 & $0,641-1,606$ \\
\hline Sim & 1 & & 1 & & 1 & \\
\hline \multicolumn{7}{|l|}{ Satisfação com o peso } \\
\hline Insatisfeito & 0,977 & $0,642-1,486$ & 0,981 & $0,725-1,329$ & 1,034 & $0,632-1,692$ \\
\hline Satisfeito & 1 & & 1 & & 1 & \\
\hline
\end{tabular}

mente significativos entre as variáveis estudadas para o sexo feminino. Para os meninos (Tabela $3)$, as variáveis escores de bens ( $\mathrm{p}$-valor $=0,003$ ) e ser elogiado pela família ( $p$-valor $=0,013$ ) apresentaram valores estatisticamente significativos para o excesso de gordura corporal. Os meninos de médio poder aquisitivo e que não eram elogiados pela família apresentavam aproximadamente 0,50 menos chance de ter excesso de gordura corporal. Com relação ao excesso de peso e à gordu- ra abdominal não houveram valores estatisticamente significativos para os meninos.

A Tabela 4 mostra os resultados da análise de regressão logística multivariados para o sexo masculino entre os maus-tratos na família e os desfechos (excesso de peso, excesso de gordura corporal e excesso de gordura abdominal). Independentemente das diferentes variáveis que poderiam influenciar a relação entre os maus-tratos e os diferentes desfechos, verificou-se que, para 
Tabela 2. Regressão logística univariada entre os desfechos e as variáveis. Adolescentes do sexo feminino. Rede Pública Municipal do Rio de Janeiro, 2007.

\begin{tabular}{|c|c|c|c|c|c|c|}
\hline \multirow{2}{*}{ Variáveis } & \multicolumn{2}{|r|}{ EP } & \multicolumn{2}{|r|}{ EG } & \multicolumn{2}{|r|}{ EGA } \\
\hline & OR & IC & OR & IC & OR & IC \\
\hline \multicolumn{7}{|l|}{ Descrição do peso } \\
\hline Abaixo do peso & $1,493^{\star}$ & $0,985-2,264$ & 1,193 & $0,813-1,752$ & 1,208 & $0,605-2,413$ \\
\hline Peso certo & 1 & & 1 & & 1 & \\
\hline Acima do peso & 1,035 & $0,677-1,583$ & $1,274^{*}$ & $0,922-1,761$ & 1,115 & $0,664-1,872$ \\
\hline \multicolumn{7}{|c|}{$\begin{array}{l}\text { Realização de alguma procedimento em } \\
\text { relação ao peso }\end{array}$} \\
\hline Sim & 0,912 & $0,652-1,278$ & 0,926 & $0,693-1,236$ & 1,257 & $0,773-2,040$ \\
\hline Não & 1 & & 1 & & 1 & \\
\hline \multicolumn{7}{|c|}{$\begin{array}{l}\text { Realização de práticas extremas para } \\
\text { controle do peso }\end{array}$} \\
\hline Sim & 0,864 & $0,406-1,840$ & 1,034 & $0,578-1,850$ & 0,599 & $0,208-1,723$ \\
\hline Não & 1 & & 1 & & 1 & \\
\hline \multicolumn{7}{|l|}{ Atividade física } \\
\hline Insuficientemente ativo & 1,218 & $0,775-1,914$ & 1,113 & $0,783-1,581$ & 1,096 & $0,577-2,083$ \\
\hline Ativo & 1 & & 1 & & 1 & \\
\hline \multicolumn{7}{|l|}{ Atividades sedentárias } \\
\hline Sedentário & 0,926 & $0,556-1,542$ & 1,038 & $0,664-1,623$ & 0,969 & $0,455-2,065$ \\
\hline Não sedentário & 1 & & 1 & & 1 & \\
\hline \multicolumn{7}{|l|}{ Agressão Física } \\
\hline Sim & $0,415^{\star * *}$ & $0,196-0,875$ & $0,690^{*}$ & $0,443-1,075$ & $0,450^{*}$ & $0,158-1,279$ \\
\hline Não & 1 & & 1 & & 1 & \\
\hline \multicolumn{7}{|l|}{ Agressão verbal } \\
\hline Sim & 0,851 & $0,566-1,280$ & 0,833 & $0,621-1,116$ & 0,731 & $0,447-1,196$ \\
\hline Não & 1 & & 1 & & 1 & \\
\hline
\end{tabular}

Tabela 3. Regressão logística univariada entre os desfechos e as variáveis. Adolescentes do sexo masculino. Rede Pública Municipal do Rio de Janeiro, 2007.

\begin{tabular}{|c|c|c|c|c|c|c|}
\hline \multirow{2}{*}{ Variáveis } & \multicolumn{2}{|r|}{ EP } & \multicolumn{2}{|c|}{ EG } & \multicolumn{2}{|c|}{ EGA } \\
\hline & OR & IC & OR & IC & OR & IC \\
\hline Idade & 1,012 & $0,859-1,193$ & 0,968 & $0,837-1,119$ & 0,948 & $0,736-1,222$ \\
\hline \multicolumn{7}{|l|}{ Cor/raça } \\
\hline Negra & 0,987 & $0,585-1,668$ & 0,850 & $0,548-1,317$ & 1,050 & $0,493-2,235$ \\
\hline Não negra & 1 & & 1 & & 1 & \\
\hline \multicolumn{7}{|l|}{ Escolaridade do chefe de família } \\
\hline Fundamental incompleto & 0,914 & $0,494-1,692$ & $0,708^{*}$ & $0,451-1,111$ & 1,160 & $0,449-2,653$ \\
\hline $\begin{array}{l}\text { Fundamental completo e médio } \\
\text { incompleto }\end{array}$ & 1,364 & $0,736-2,528$ & 1,026 & $0,667-1,579$ & 1,500 & $0,583-3,861$ \\
\hline Médio incompleto & 1 & & 1 & & 1 & \\
\hline \multicolumn{7}{|l|}{ Escores de bens } \\
\hline Baixo poder aquisitivo & 1,124 & $0,659-1,920$ & $0,727^{\star}$ & $0,486-1,087$ & 0,757 & $0,356-1,610$ \\
\hline Médio poder aquisitivo & 0,935 & $0,563-1,554$ & $0,550^{\star * *}$ & $0,369-0,819$ & $0,610^{\star}$ & $0,304-1,225$ \\
\hline Alto poder aquisitivo & 1 & & 1 & & 1 & \\
\hline \multicolumn{7}{|l|}{ Elogiado pela família } \\
\hline Não & 1,141 & $0,672-1,938$ & $0,498^{\star * *}$ & $0,288-0,859$ & $0,336^{\star *}$ & $0,099-1,134$ \\
\hline Sim & 1 & & 1 & & 1 & \\
\hline
\end{tabular}


Tabela 3. Regressão logística univariada entre os desfechos e as variáveis. Adolescentes do sexo masculino. Rede Pública Municipal do Rio de Janeiro, 2007.

\begin{tabular}{|c|c|c|c|c|c|c|}
\hline \multirow{2}{*}{ Variáveis } & \multicolumn{2}{|r|}{ EP } & \multicolumn{2}{|r|}{ EG } & \multicolumn{2}{|c|}{ EGA } \\
\hline & OR & IC & OR & IC & OR & IC \\
\hline \multicolumn{7}{|l|}{ Família ciente do tempo livre } \\
\hline Não & 1,161 & $0,716-1,882$ & $0,713^{* *}$ & $0,485-1,049$ & 0,878 & $0,446-1,728$ \\
\hline $\operatorname{Sim}$ & 1 & & 1 & & 1 & \\
\hline \multicolumn{7}{|l|}{ Pais cientes de faltar aula } \\
\hline Não & 0,828 & $0,550-1,245$ & 0,984 & $0,682-1,419$ & 0,936 & $0,496-1,767$ \\
\hline Sim & 1 & & 1 & & 1 & \\
\hline \multicolumn{7}{|c|}{$\begin{array}{l}\text { Pais se importam com uso de álcool/ } \\
\text { fumo/drogas }\end{array}$} \\
\hline Não se importam & 0,836 & $0,382-1,832$ & 0,846 & $0,480-1,492$ & 1,368 & $0,520-3,598$ \\
\hline Se importam & 1 & & 1 & & 1 & \\
\hline \multicolumn{7}{|l|}{ Boa relação com colega } \\
\hline Não & 1,094 & $0,510-2,346$ & $0,602^{*}$ & $0,322-1,123$ & 0,511 & $0,114-2,296$ \\
\hline Sim & 1 & & 1 & & 1 & \\
\hline \multicolumn{7}{|l|}{ Diálogo com o professor } \\
\hline Não & 0,827 & $0,543-1,258$ & 1,032 & $0,740-1,440$ & $0,652^{*}$ & $0,351-1,211$ \\
\hline Sim & 1 & & 1 & & 1 & \\
\hline \multicolumn{7}{|l|}{ Satisfação com o peso } \\
\hline Insatisfeito & 1,150 & $0,723-1,830$ & 1,152 & $0,765-1,737$ & 1,133 & $0,576-2,228$ \\
\hline Satisfeito & 1 & & 1 & & 1 & \\
\hline \multicolumn{7}{|l|}{ Descrição do peso } \\
\hline Abaixo do peso & 0,956 & $0,605-1,512$ & 0,927 & $0,648-1,326$ & 0,927 & $0,453-1,896$ \\
\hline Peso certo & 1 & & 1 & & 1 & \\
\hline Acima do peso & 1,333 & $0,818-2,171$ & $1,404^{*}$ & $0,915-2,152$ & $1,824^{*}$ & $0,874-3,812$ \\
\hline \multicolumn{7}{|c|}{$\begin{array}{l}\text { Realização de algum procedimento em } \\
\text { relação ao peso }\end{array}$} \\
\hline Sim & $0,716^{*}$ & $0,461-1,114$ & 1,125 & $0,803-1,575$ & 1,240 & $0,642-2,393$ \\
\hline Não & 1 & & 1 & & 1 & \\
\hline \multicolumn{7}{|c|}{$\begin{array}{l}\text { Realização de práticas extremas para } \\
\text { controle do peso }\end{array}$} \\
\hline Sim & 0,543 & $0,168-1,756$ & $0,534^{*}$ & $0,215-1,325$ & 0,515 & $0,066-4,009$ \\
\hline Não & 1 & & 1 & & 1 & \\
\hline \multicolumn{7}{|l|}{ Atividade física } \\
\hline Insuficientemente ativo & 0,808 & $0,515-1,268$ & $0,794^{*}$ & $0,576-1,095$ & 0,716 & $0,383-1,339$ \\
\hline Ativo & 1 & & 1 & & 1 & \\
\hline \multicolumn{7}{|l|}{ Atividades sedentárias } \\
\hline Sedentário & 1,547 & $0,676-3,541$ & 1,290 & $0,726-2,294$ & 0,928 & $0,316-2,726$ \\
\hline Não sedentário & 1 & & 1 & & 1 & \\
\hline \multicolumn{7}{|l|}{ Agressão Física } \\
\hline Sim & 1,150 & $0,532-2,485$ & 1,243 & $0,700-2,206$ & 1,079 & $0,320-3,637$ \\
\hline Não & 1 & & 1 & & 1 & \\
\hline \multicolumn{7}{|l|}{ Agressão verbal } \\
\hline Sim & 1,234 & $0,813-1,873$ & 1,174 & $0,794-1,736$ & 1,146 & $0,598-2,194$ \\
\hline Não & 1 & & 1 & & 1 & \\
\hline
\end{tabular}


Tabela 4. Modelos de regressão logística ajustada entre os maus-tratos familiares e os desfechos segundo o sexo. Rede Pública Municipal do Rio de Janeiro, 2007.

\begin{tabular}{|c|c|c|c|c|c|c|}
\hline \multirow{2}{*}{$\begin{array}{l}\text { Maus-tratos } \\
\text { familiares }\end{array}$} & \multicolumn{2}{|c|}{ EP } & \multicolumn{2}{|c|}{ EG } & \multicolumn{2}{|c|}{ EGA } \\
\hline & OR & IC & OR & IC & OR & IC \\
\hline & \multicolumn{6}{|c|}{ Sexo Feminino } \\
\hline Agressão Física & $0,465^{\star * *}$ & $0,220-0,983$ & $0,672^{\star}$ & $0,416-1,086$ & $0,506^{*}$ & $0,178-1,437$ \\
\hline \multirow[t]{2}{*}{ Agressão verbal } & 0,940 & $0,606-1,458$ & 0,827 & $0,598-1,144$ & 0,716 & $0,428-1,200$ \\
\hline & \multicolumn{6}{|c|}{ Sexo Masculino } \\
\hline Agressão Física & 1,149 & $0,523-2,526$ & 1,488 & $0,789-2,808$ & 1,168 & $0,347-3,932$ \\
\hline Agressão verbal & 1,227 & $0,808-1,864$ & 1,209 & $0,775-1,886$ & 1,280 & $0,650-2,520$ \\
\hline \multicolumn{7}{|c|}{$\begin{array}{l}{ }^{*} \mathrm{p} \text {-valor }<0,20,{ }^{* *} \mathrm{p} \text {-valor }<0,10,{ }^{* *} \mathrm{p} \text {-valor }<0,05 . \mathrm{OR}-\mathrm{Odds} \text { Ratio; IC -Intervalo de confiança; EP - Excesso de peso; EG - } \\
\text { Excesso de gordura corporal; EGA - Excesso de gordura abdominal. }\end{array}$} \\
\hline \multicolumn{7}{|c|}{$\begin{array}{l}\text { Para o EP, os modelos foram ajustados pelas variáveis: 1) escores de bens, escolaridade do chefe de família e descrição do peso } \\
\text { - sexo feminino, e 2) Realização de algum procedimento em relação ao peso - sexo masculino. Para o EG, os modelos foram } \\
\text { ajustados pelas variáveis: 1) escolaridade do chefe de família, descrição do peso, pais cientes do tempo livre e pais cientes de faltar } \\
\text { aula - sexo feminino, e 2) escolaridade do chefe de família, escores de bens, pais cientes do tempo livre, elogiado pela família, } \\
\text { relação com o colega, descrição do peso, atividade física e realização de práticas extremas para controle de peso - sexo masculino. } \\
\text { Para o EGA, os modelos foram ajustados pelas variáveis: 1) escolaridade do chefe de família e relação com o colega - sexo } \\
\text { feminino, e 2) escores de bens, diálogo com o professor, elogiado pela família e descrição do peso - sexo masculino. }\end{array}$} \\
\hline
\end{tabular}

os meninos, não houve associação significativa entre os dois tipos de maus-tratos e os desfechos, mas foi observado que, na presença deles, existe uma tendência de maior ocorrência de alteração na composição corporal convergindo para o excesso, apesar de tais associações não serem estatisticamente significativas.

Em contrapartida, as meninas diferiram dos meninos. Foi observado que tanto nas análises brutas (Tabela 2) quanto nas ajustadas para escolaridade do chefe de família, escores de bens e descrição de peso (Tabela 4), a agressão física se apresentava como um fator associado à ocorrência do excesso de peso, mas de forma inversa. $\mathrm{Na}$ presença de agressão física, as meninas apresentaram cerca de $50 \%$ menos chance de ter excesso de peso $(\mathrm{OR}=0,465 ; \mathrm{IC}=0,220-0,983)$. Esses valores também seguiram o mesmo padrão para o excesso de gordura corporal $(\mathrm{OR}=0,7)$ e o excesso de gordura abdominal $(\mathrm{OR}=0,5)$, apesar desta associação não ser significativa estatisticamente. Para a agressão verbal, os resultados apontam na mesma direção apesar de as associações não serem estatisticamente significativas.

\section{Discussão}

Os resultados do presente estudo mostraram que os maus-tratos familiares não se associaram de forma positiva ao excesso de peso, ao excesso de gordura corporal e abdominal. Entretanto, as meninas que sofreram agressão física tinham menos chances de apresentar excesso de peso. Res- salta-se que, essas análises foram ajustadas para as variáveis: relação com a família e com a escola, nível de atividade física e sedentarismo, condição de peso, satisfação corporal e práticas extremas para controle de peso que, segundo a literatura, são variáveis associadas ao excesso de peso ${ }^{29,30}$.

Esse achado difere dos expostos em outros estudos. Danese e $\operatorname{Tan}^{16}$, por exemplo, em revisão sistemática e metanálise sobre o assunto, verificaram que para o grupo de crianças e adolescentes a associação entre os maus-tratos familiares e o excesso de peso não foi verificada $(\mathrm{OR}=1,3$; IC $=0,92-1,39)$. Vale ressaltar que os autores não procederam às análises segundo sexo, questão importante a ser considerada em pesquisas com adolescentes. Nesse período de vida, os mecanismos de defesa ao trauma (distorção do comportamento alimentar) ou do aumento de peso são diferentes entre o sexo feminino e o masculino ${ }^{31}$ existe um maior ganho de massa muscular para os meninos e de tecido de gordura para as meninas $^{32}$. Faz-se necessário destacar que, na referida revisão, os autores apresentam estudos que usaram índices/indicadores antropométricos diferentes dos utilizados no presente estudo, o que dificulta a comparabilidade entre resultados.

Em relação aos estudos com adolescentes que apresentaram seus resultados de acordo com o sexo e abordam os maus-tratos físico e mental/ emocional/verbal e o excesso de peso, notou-se que, da mesma forma que ocorreu no presente estudo, outras pesquisas sobre o assunto apresentam resultados distintos entre os sexos. Neste trabalho, não houve associação entre os maus-tratos 
familiares e o excesso de peso para os meninos, já para as meninas vítimas de agressão física, sim. $\mathrm{Na}$ esfera internacional, Stensland et al. ${ }^{30}$ observaram que a exposição a vários tipos de violência (física, verbal, sexual) gerava mais altos IMC em adolescentes noruegueses de 12 a 19 anos, mas esses valores apresentavam-se menos evidentes no sexo masculino. Entretanto, para Jun et al. ${ }^{33}$ em uma coorte com adolescentes americanos de 12 a 20 anos, os meninos expostos a violência doméstica (física, emocional ou sexual) exibiam associação com o desenvolvimento e a manutenção da obesidade, enquanto as meninas não. De acordo com Veldwijk et al. ${ }^{31}$, em um estudo com escolares holandeses de 13 a 16 anos, tanto os meninos quanto as meninas eram mais propensos ao sobrepeso e à obesidade na presença do abuso mental, enquanto a ocorrência de abuso físico e sexual era associada à obesidade somente para os meninos.

Também se observa diferença quanto à direção das associações. Schneiderman et al. ${ }^{34}$, em um estudo sobre a violência física, emocional, sexual e a negligência com crianças e adolescentes de Los Angeles de 9 a 12 anos, verificaram uma associação inversa entre a violência e o excesso de peso (IMC $\geq$ percentil 85 ). As meninas vítimas de abuso físico possuíam menores chances de apresentar excesso de peso quando comparadas àquelas que não sofreram abuso físico $(\mathrm{OR}=0,29$; IC95\% $=0,10-0,87)$. Vale ressaltar que tanto este estudo como o de Schneiderman et al. ${ }^{34}$, os quais são de corte transversal, utilizaram categorias de IMC para avaliar o excesso de peso em adolescentes e verificaram a existência de associação negativa entre a violência física e a categoria de IMC que engloba o sobrepeso e a obesidade somente para o sexo feminino.

Já no âmbito nacional, Silva et al. ${ }^{15}$ estudando adolescentes de 10 a 19 anos de uma unidade de saúde do município do Rio de Janeiro observaram uma relação entre a violência familiar e o IMC somente para as meninas. As adolescentes vítimas de agressão verbal pelos pais apresentavam aumentos significativos de IMC. De forma distinta ao presente estudo, os autores relataram uma relação positiva entre maus-tratos e o IMC para o sexo feminino. Por outro lado, Gagliano ${ }^{14}$, ao pesquisar escolares do $1^{\circ}$ ano do ensino médio de escolas públicas e privadas do estado do Rio de Janeiro, verificou que não existia uma relação positiva entre os maus-tratos (negligência, abuso físico e abuso emocional) para ambos os sexos, mas notou uma redução de IMC para os meninos vítimas de negligência física $(\beta=-0,196$; IC95\% $=-0,346-0,045)$.
Assim, nem sempre as vítimas de maus-tratos apresentam excesso de peso. Uma parte das pesquisas mostra que a associação tende para uma direção inversa, ou seja, a violência estaria atrelada à redução ou valores mais baixos de $\mathrm{IMC}^{14,34}$, conforme o observado neste estudo.

No que tange às diferenças entre os sexos encontradas neste estudo e na literatura, é importante destacar que existem diferenças de resposta entre os indivíduos com relação às adversidades e ao potencial de resiliência que muitas vítimas podem apresentar ${ }^{35}$. Compreender as diferenças entre os gêneros ${ }^{34}$ talvez seja um caminho a ser explorado para tentar explicar o resultado encontrado no presente estudo. Seguindo esse raciocínio, nas mulheres o trauma pode ser contrabalanceado por influências sociais para ser magra e isso pode ser mais evidente durante o período da adolescência. É observado neste estudo que $31,5 \%$ das meninas apresentavam insatisfação corporal, o que pode gerar comportamentos compensatórios para se atingir o ideal corporal imaginado por elas. Assim, as adolescentes sob influência do ideal de magreza postulado pela sociedade e pela mídia não desenvolveriam excesso de peso mesmo na presença de maus-tratos, como também elas seriam mais vulneráveis a algum distúrbio alimentar ou mesmo teriam medo de comer para não perder o controle ${ }^{29}$. Talvez essa seja uma possível explicação para a ocorrência da associação inversa encontrada no presente estudo. Ao analisarmos essa perspectiva, foi observado que os maus-tratos estavam associados à insatisfação com o peso no grupo das meninas (OR = 1,69; IC = 1,24-2,31), como também se associavam ao uso de práticas extremas de controle de peso $(\mathrm{OR}=1,83$; $\mathrm{IC}=1,03-3,25)$ (dados não apresentados). Pode-se supor que as meninas vítimas de maus-tratos apresentavam maiores chances de relatar uma insatisfação corporal e algum mecanismo extremo para controle de peso e isso possivelmente poderia refletir em menores chances de se ter excesso de peso.

Com relação à mensuração dos maus-tratos familiares, observa-se na literatura o uso de diferentes índices/indicadores/medidas/instrumentos para aferi-los ${ }^{14,15,31,34,36}$. Isso pode dificultar a comparabilidade dos resultados encontrados entre os estudos, porém, de forma similar ao presente estudo, Hussey et al. e Veldwijk et al. investigaram um determinado tipo de maus-tratos por meio de uma única pergunta. Hussey et al. ${ }^{36}$ avaliaram a agressão física com respostas relacionadas à frequência que este abuso acontecia, e posteriormente as respostas foram categorizadas 
em nunca (quando ausente) e uma ou mais vezes (quando presente). Veldwijk et al. ${ }^{31}$, além da agressão física, aferiram também o abuso emocional. E de maneira similar, utilizaram somente uma questão para caracterizar cada um dos abusos/agressões explorados. Cabe ainda mencionar que, assim como no presente estudo, os autores verificaram a associação entre os maus-tratos e o estado de peso/IMC por meio de um estudo de corte transversal, desenhado originalmente para monitorar a saúde, bem-estar e estilo de vida dos adolescentes.

Assim como os resultados de outras pesquisas $^{14,34}$, os achados deste estudo mostraram que existe associação negativa entre os maus-tratos e o excesso de peso (IMC > percentil $85^{29,34,36}$ ). Vale ressaltar que, embora o IMC não faça distinção entre o tecido livre de gordura e a massa livre de gordura corporal ${ }^{37}$, ele é um índice recomendado para definir o excesso de peso em estudos epiodemiológicos ${ }^{38}$, pois é um importante indicador de prognóstico de saúde, sendo capaz de identificar adolescentes em situação de risco de diabetes e doenças cardíacas ${ }^{39}$. Adicionalmente, pode-se pôr em evidência que neste artigo buscou-se conhecer um pouco mais a relação da violência com a composição corporal, explorando-se também a associação entre maus-tratos familiares e a RCA e o percentual de gordura - padrão de distribuição de gordura. Destaca-se que investigações mostraram que indivíduos com gordura abdominal apresentam maior risco para problemas de saúde do que aqueles com depósito de gordura distribuídos perifericamente ${ }^{8,39}$. Conforme pode ser observado na Tabela 4, as associações entre os maus-tratos familiares e o excesso de peso e de gordura apontam para uma direção positiva no sexo masculino e para uma negativa no sexo feminino, independente do índice antropométrico utilizado. Embora não sejam significativos os achados relativos à RCA e ao percentual de gordura, assemelham-se ao encontrado com IMC, o que indica que mais estudos com intuito de esclarecer a dinâmica destas associações merecem ser implementados.

Uma limitação deste estudo foi o uso de uma única pergunta para se medir, definir e classificar os dois tipos de maus-tratos (agressão física e verbal), mas como já observado na literatura outros autores também o fizeram por esse modo ${ }^{31}$. Com relação à pergunta maus-tratos (agressão verbal/ abuso emocional), Tricket et al. ${ }^{40}$ relataram que o abuso emocional apresenta categorias com relação ao comportamento dos pais que descrevem esse tipo de abuso por meio do rejeitar, aterrorizar, isolar, explorar/corromper. Vários comporta- mentos podem ser relatados para cada uma dessas categorias, mas nem todos os autores utilizam todas elas para avaliar o abuso emocional ${ }^{31,40}$. Isso também ocorreu neste estudo, já que os dados analisados foram de um inquérito realizado com os escolares do município do Rio, o qual somente contemplou a agressão verbal por meio da pergunta: "Nos últimos 30 dias, com que frequência sua família te esculachou (tratou muito mal)?”.

A pergunta pertinente a agressão física “... quantas vezes foi agredido fisicamente por um adulto da família?", não esclarece especificamente o que seria ser agredido fisicamente. Diferente das perguntas usadas por Goodwin e Wamdoldt: "Sua mãe ou pai ... chutou ou bateu em você com o punho, bateu ou tentou bater com alguma coisa, bateu em você, sufocou, queimou ou escaldou você..." ${ }^{41}$, que detalharam os atos considerados por eles como agressão física. Nessa perspectiva, é possível que por não terem sido mencionados os atos da agressão física, o presente estudo somente tenha registrado os casos mais graves que de acordo com a literatura são os mais lembra$\operatorname{dos}^{42}$ e, o que pode, portanto, ter subestimado os resultados das exposições investigadas.

Uma outra limitação seria a natureza do desenho do estudo - transversal. Uma única observação em um determinado período de tempo pode não refletir a ocorrência do excesso de peso e de gordura que porventura possa acometer os indivíduos no decorrer do seu crescimento e desenvolvimento. Dessa forma, este tipo de estudo é incapaz de fazer inferência causal ${ }^{11}$.

O ponto forte deste estudo foi a utilização de medidas antropométricas de peso, altura, perímetro abdominal e dobras cutâneas para avaliar o estado nutricional por meio de três índices antropométricos. Essas medidas foram realizadas por uma equipe treinada fornecendo, portanto, estimativas confiáveis e válidas sobre a composição corporal dos indivíduos. Isso possibilitou classificar os adolescentes para excesso de peso, de gordura corporal e de gordura abdominal, tornando este estudo pioneiro nessa abordagem. O presente estudo apresentou uma abordagem diferenciada quando também investigou a relação entre os maus-tratos familiares e o excesso de gordura corporal e de gordura abdominal, aspecto importante para os adolescentes, uma vez que durante o crescimento mudanças na composição corporal podem se refletir tanto em ganho de gordura corporal quanto em massa livre de gordura ${ }^{32}$.

Em suma, os resultados do presente estudo mostraram que nenhum dos dois tipos de maustratos investigados se associou de maneira esta- 
tisticamente significativa ao excesso de peso, de gordura corporal e de gordura abdominal nos meninos. Já em relação às meninas foi observado que aquelas que sofreram agressão física eram menos propensas a apresentar IMC > percentil $85(\mathrm{RC}=0,499 ; \mathrm{IC}=0,212-0,951)$.

Apesar das limitações, este estudo possibilitou a exploração de um tema pouco investigado na adolescência, principalmente no que se refere ao excesso de gordura. Os achados desta investigação podem servir de alerta para a comunidade científica e para as redes de saúde e de educação do nosso país. O conhecimento da existência da relação violência-excesso de peso e de gordura durante a adolescência, particularmente no sexo feminino, permite que novas estratégicas de ação sejam implementadas. Interessante seria ainda o exercício de uma reflexão acerca dos potenciais mecanismos que podem levar ao aumento ou à redução do peso e de gordura em resposta aos maus-tratos, onde as diferenças entre os gêneros estejam presentes.

\section{Colaboradores}

AMVL Silva trabalhou na análise e interpretação dos dados e redação do artigo e MH Hasselmann na revisão crítica do conteúdo intelectual. Todos os autores revisaram a versão final do manuscrito e aprovaram para a publicação.

\section{Referências}

1. CDC. Overweight and Obesity. Atlanta: CDC; 2014 [cited 2015 Mai 18]. Available from: http://www.cdc.gov/ obesity/childhood/index.html

2. Instituto Brasileiro de Geografia e Estatística (IBGE). Pesquisa de Orçamentos Familiares 2008-2009: Antropometria e Estado Nutricional de crianças, adolescentes e adultos no Brasil. Rio de Janeiro: IBGE; 2010 [cited 2013 May 20]. Available from: http://ibge.gov.br/home/estatistica/populacao/condicaodevida/pof/2008_2009_encaa/pof_20082009_encaa.pdf.

3. Instituto Brasileiro de Geografia e Estatística (IBGE). Pesquisa Nacional de Saúde do Escolar (Pense) 2009. Rio de Janerio: IBGE; 2009.

4. Santana JCB, Pitrez Filho MLS, Vargas LTR, Hauschild jA, Pinto LA, Pitrez EH, Bruscato NM, Oliveira JR, Moriguchi E. Relação entre medidas antropométricas em adolescentes e fatores de risco cardiometabólicos em adultos jovens. Acta Pediatr Port 2012; 43(6):225232.

5. Fontana PV, Giannini DT. Associação de indicadores antropométricos com fatores de risco cardiovascular em adolescentes com excesso de peso. Adolesc Saude 2014; 11(4):68-78.

6. Kondaki K, Grammatikaki E, Pavón DJ, Manios Y, González-Gross M, Sjöstrom M, Gottrand F, Molnar D, Moreno LA, Kafatos A, Gilbert C, Kersting M, De Henauw S. Comparison of several anthropometric indices with insulin resistance proxy measures among European adolescents: The Helena Study. Eur J Pediatr 2011; 170(6):731-739.

7. Stabelini Neto A, Bozza R, Ulbrich A, Mascarenhas LPG, Boguszewski MCS, Campos W. Síndrome metabólica em adolescentes de diferentes estados nutricionais. Arq Bras Endocrionol Metab 2012; 56(2):104-109.
8. Botton J, Heude B, Kettaneh A, Borys JM, Lommez A, Bresson JL, Ducimetiere P, Charles MA; FLVS Study Group. Cardiovascular risk factor levels and their relationships with overweight and fat distribution in children: the Fleurbaix Laventie Ville Sante II study. Metabolism: clinical and experimental 2007; 56(5):614-622.

9. Schraiber L. Violência vivida: A dor que não tem nome. Interface (Botucatu) 2003; 5(10):41-54.

10. Martins CBG, Mello Jorge MHP. A violência contra crianças e adolescentes: características epidemiológicas dos casos notificados aos Conselhos Tutelares e programas de atendimento em município do Sul do Brasil, 2002 e 2006. Epidemiol Serv Saúde 2009; 18(4):315-334.

11. Midei AJ, Matthews KA. Interpersonal violence in childhood as a risk factor for obesity: a systematic review of the literature and proposed pathways. Obes Rev 2011; 12(5):e159-172.

12. Norman RE, Byambaa M, De R, Butchart A, Scott J, Vos T. The Long-Term Health Consequences of Child PhysicalAbuse, Emotional Abuse, and Neglect: A Systematic Review and Meta-Analysis. PLOS Medicine 2012; 9(11):e1001349.

13. Shaw NJ, Crabtree NJ, Mohammed SK, Fordham JN. Ethnic and gender differences in body fat in British schoolchildren as measured by DXA. Arch Dis Child 2007; 92:872-875.

14. Gagliano AHNP. Violência familiar na infância: fator de risco para o excesso de peso em adolescentes? [tese ]. Rio de Janeiro: Universidado do Estado do Rio de Janeiro; 2012.

15. Silva AMVL, Taquette SR, Hasselmann MH. Family violence and body mass index among adolescents enrolled in the Bolsa Família Program and treated at a primary care clinic. Cad Saude Publica 2014; 30(3):645-655. 
16. Danese A, Tan M. Childhood maltreatment and obesity: systematic review and meta-analysis. Molecular Psychiatry 2014; 19(5):544-554.

17. Jerônimo DP, Miranda H, Souza RA, Pinto MC. Estudo descritivo da gordura corporal predita por métodos antropométricos duplamente indiretos em escolares de 11 a 14 anos. Revista Brasileira de Obesidade, Nutrição e Emagrecimento 2009; 3(14):88-93.

18. Beck CC, Lopes AS, Pitanga FJG. Indicadores antropométricos de sobrepeso e obesidade como preditores de alterações lipídicas em adolescentes. Rev Paul Pediatr $2011 ; 29(1): 46-53$.

19. Castro IRR, Cardoso LO, Engstrom EM, Levy RB, Monteiro CA. Vigilância de fatores de risco para doenças não transmissíveis entre adolescentes: a experiência da cidade do Rio de Janeiro, Brasil. Cad Saude Publica 2008; 24(10):2279-2288.

20. Cardoso LO. Fatores associados ao excesso de peso e perfis de consumo e comportamento alimentar de adolescentes [tese]. Rio de Janeiro: Fundação Oswaldo Cruz; 2010.

21. World Health Organization (WHO). Physical Status: The use and interpretation of Anthropometry. Geneva: WHO; 1995.

22. Lohman TG, Roche AF, Martorell R. Anthropometric Standardization Reference Manual. Champaign: Human Kinetics Books; 1988.

23. Habitch JP. Estandarizacion de métodos epidemiológicos cuantitativos sobre el terreno. Bol Oficina Sanit Panam 1974; 76(5):375-384.

24. World Health Organization (WHO). Growth reference data for 5-19 years 2007. [cited 2008 Jun 20]. Available from: http://www.who.int/growthref/en/

25. Slaughter MH, Lohman TG, Boileau BA, Horswill CA, Stillman RJ, Van Loan MD, Bemben DA. Skinfold Equations for Estimation of Body Fatness in Children and Youth. Human Biology 1998; 60(5):709-723.

26. Lohman TG. The use of skinfold to estimate body fitness on children and youth. Journal of physical education, recreation \& dance 1987; 58(9):98-103.

27. American Academy of Pediatrics. Children, Adolescents, and the Media. Council on Comunnications and Media. Pediatrics 2013; 132(5):958.

28. Barufaldi LA, Abreu GA, Coutinho ESF, Bloch KV. Meta-analysis of the prevalence of physical inactivity among Brazilian adolescents. Cad Saude Publica 2012; 28(6):1019-1032.

29. Fuemmeler BF, Dedert E, McClernon FJ, Beckham JC. Adverse childhood events are associated with obesity and disordered eating: results from a U.S. population-based survey of young adults. J Trauma Stress 2009; 22(4):329-333.

30. Stensland SO, Thoresen S, Wentzel-Larsen T, Dyb G. Interpersonal violence and overweight in adolescents: the HUNT Study. Scand J Public Health 2015; 43(1):1826.

31. Veldwijk J, Proper KI, Hoeven-Mulder HB, Bemelmans WJ. The prevalence of physical, sexual and mental abuse among adolescents and the association with BMI status. BMC Public Health 2012; 12(840):1-9.
32. Barbosa KBF, Franceschini SDCC, Priore SE. Influência dos estágios de maturação sexual no estado nutricional, antropometria e composição corporal de adolescentes. Rev Bras Saúde Matern Infant 2006; 6(4):375-382.

33. Jun H-J, Corliss HL, Boynton-Jarrett Re, Spiegelman D, Austin SB, Wright RJ. Growing up in a domestic violence environment: relationship with developmental trajectories of body mass index during adolescence into young adulthood. J Epidemiol Community Health 2012; 66(7):629-635.

34. Schneiderman JU, Mennen FE, Negriff S, Trickett PK. Overweight and obesity among maltreated young adolescents. Child Abuse Negl 2012; 36(4):370-378.

35. Noll JG, Zeller MH, Trickett PK, Putnam FW. Obesity risk for female victims of childhood sexual abuse: a prospective study. Pediatrics 2007; 120(1):e61-67.

36. Hussey JM, Chang JJ, Kotch JB. Child maltreatment in the United States: prevalence, risk factors, and adolescent health consequences. Pediatrics 2006; 118(3):933942.

37. Brambilla P, Bedogni G, Heo M, Pietrobelli A. Waist circumference-to-height ratio predicts adiposity better than body mass index in children and adolescents. Int $J$ Obes (Lond) 2013; 37(7):943-946.

38. Bluher S, Meigen C, Gausche R, Keller E, Pfaffle R, Sabin M, Werther G, Odeh R, Kiess W. Age-specific stabilization in obesity prevalence in German children: a cross-sectional study from 1999 to 2008. Int J Pediatr Obes 2011; 6(2-2):e199-206.

39. Browning LM, Hsieh SD, Ashwell M. A systematic review of waist-to-height ratio as a screening tool for the prediction of cardiovascular disease and diabetes: 0.5 could be a suitable global boundary value. Nutrition research reviews 2010; 23(2):247-269.

40. Trickett PK, Mennen FE, Kim K, Sang J. Emotional abuse in a sample of multiply maltreated, urban young adolescents: Issues of definition and identification. Child Abuse \& Neglect 2009; 33(1):27-35.

41. Goodwin RD, Wamboldt FS. Childhood physical abuse and respiratory disease in the community: the role of mental health and cigarette smoking. Nicotine \& tobacco research: official journal of the Society for Research on Nicotine and Tobacco 2012; 14(1):91-97.

42. Straus MA, Gelles JR. Physical Violence in American Families: Risk Factors and Adaptations to Violence in 8,145 Families. New Brunswick: Transaction Publishers; 1995.

Artigo apresentado em 02/02/2016

Aprovado em 07/12/2016

Versão final apresentada em 09/12/2016 